\title{
Aplicação da metodologia da assistência a pacientes com Lúpus Eritematoso Sistêmico em pulsoterapia: uma experiência docente
}

\author{
Application of assistance methodology in patients suffering Systemic Erythematosus \\ Lupus in drug pulse therapy: a teaching experience \\ Uso de la metodología de la asistencia a pacientes con Lupus Eritematoso Sistémico \\ en quimioterapia por pulso: una experiencia de enseñanza
}

\section{Maria Gorette dos Reis \\ Marisa Dias Rolan Loureiro \\ Maria da Graça da Silva}

Enfermeiras. Mestres em Enfermagem. Docentes do Departamento de Enfermagem da Universidade Federal de Mato Grosso do Sul, Campo Grande, MS.
Submissão: $18 / 10 / 2005$ Aprovação: $15 / 08 / 2006$

\section{RESUMO}

Este trabalho é um relato de experiência do ensino de Enfermagem Clínica para alunos de graduação, no qual foi utilizada a metodologia de assistência de enfermagem como estratégia de ensino. Aplicada a pessoas adultas, portadoras de Lúpus Eritematoso Sistêmico (LES), em sua maioria mulheres jovens, submetidas a pulsoterapia, uma modalidade terapêutica que, por meio da imunossupressão, reduz 0 processo inflamatório causado pelos autoanticorpos. Desta forma, a assistência é sistematizada, individualizada e integral e permite ao acadêmico, oportunidades singulares para correlação teóricoprática, com a aplicação de conhecimentos básicos da área de formação, contextualização do processo saúde-doença e focalização ampliada para além do biológico, abrangendo aspectos emocionais, sociais e espirituais e oportunidade para o aluno avaliar as diversas nuanças que interferem nas condições de assistência oferecidas pelos serviços públicos de saúde.

Descritores: Pulsoterapia; Prestação de Cuidados de Saúde, Lúpus Eritematoso Sistêmico.

\section{ABSTRACT}

This research is an experience report of clinical teaching for undergraduate nursing students, to whom it was used the assistance methodology as a teaching strategy. It was applied in adults, sufferingSystemic Erythematosus Lupus, young women, in majority, who were submitted to drug pulse therapy, a therapeutic modality which, through immunesuppression, reduces the inflammatory process caused by selfantibody. This way, the assistance is systemized, individualized and integral and it allows single opportunities of theoric-pratic correlation for the students, with the application of basic knowledge of the formation area, context of the health-disease process and the focus going beyond the biologic, including emotional, social and spiritual aspects and opportunities for the student to evaluate several factors that interfere in the assistance conditions offered by public health services.

Descriptors: Pulse therapy, drug; Delivery of health care; Lupus Erythematosus, Systemic.

\section{RESUMEN}

Este és un relato de experimento de la enseñanza de Enfermería Clínica para alumnos de graduación, utilizando medología de asistencia de enfermería como habilidad medotológica para acompañar el paciente y enseñar. Empleada a la personas adultas, portadoras de Lúpus Eritematoso Sistémico (LES), en su mayoría mujeres jóvenes, sometidas a la pulsoterapia, una modalidad terapéutica que, por medio de la imunosupresión, reducido el proceso inflamatorio causante por los autoanticuerpos. De esta manera, la asistencia és de sistematización, particular y total, concede al académico, ocasiones raras para correlación teoríca-práctica, con la aplicación del conocimiento básico de la área de formación, contextualización del proceso salud-enfermedad y focalización ampliada para más adelante del biológico, abarcando aspectos emocionales, sociales y espirituales y ocasión para el alumno evaluar las diversas matizes que interponen en las condiciones de asisténcia ofrecidas por los servícios públicos de salud. Descriptores: Quimioterapia por pulso; Prestación de atención de salud; Lupus Eritematoso Sistêmico.

Reis MG, Loureiro MDR, Silva MG. Aplicação da metodologia da assistência a pacientes com Lúpus Eritematoso Sistêmico em pulsoterapia: uma experiência docente. Rev Bras Enferm 2007 mar-abr; 60(2):229-32.

\section{INTRODUÇÃO}

Desde 1991, quando da criação do curso de graduação em Enfermagem na Universidade Federal de Mato Grosso do Sul (UFMS), nós, as docentes da disciplina de Enfermagem Clínica temos buscado subsídios para ministrar os conteúdos teórico-práticos utilizando princípios da Metodologia daAssistência, através da aplicação do Processo de Enfermagem, utilizando um roteiro elaborado para tal fim. Citando Robazzi et a ${ }^{(1)}$, encontram-se empenhadas em ensinar uma metodologia composta das fases de: coleta de 
dados, elaboração de Diagnósticos de Enfermagem (segundo a Taxonomia preconizada pela North American Nursing Diagnosis Association - NANDA, planejamento da assistência, implementação e avaliação da mesma.

Concordamos com as autoras citadas, quando dizem ser necessário, para elaboração de diagnóstico, além do relacionamento terapêutico enfermagem/paciente, o conhecimento integral do mesmo, incluindo fisiopatologia.

Dentre as várias funções da Enfermagem, em especial do enfermeiro, destacamos a realização de diagnósticos de reações que requeiram intervenções de enfermagem, detectando problemas reais ou potenciais. 0 Processo de Enfermagem é o método através do qual o conhecimento teórico é aplicado à prática, exigindo habilidades cognitivas, técnicas e interpessoais(2).

Considerando a elevada incidência de Lupus Eritematoso Sistêmico (LES) no setor de Clínica Médica do Núcleo de Hospital Universitário UFMS, local que é campo de atividades práticas da disciplina de Enfermagem Clínica, tal conteúdo torna-se prioritário.

O maior número de casos de LES ocorre entre mulheres jovens (entre 20 e 40 anos), na proporção de 9 a 10 mulheres para um homem, em todo 0 mundo e em todas as raças ${ }^{(3,4)}$, o que justifica a predominância de pacientes femininas, em idade reprodutiva, internadas no referido hospital.

O LES pode ser definido como uma doença de natureza auto-imune, crônica, inflamatória, de etiologia desconhecida, que afeta múltiplos órgãos, com sintomas localizados e sistêmicos. Caracteriza-se por períodos de remissão e exacerbação das manifestações clínicas, alterações do colágeno e lesões vasculares causadas por ação de auto-anticorpos, os quais são mediadores imunológicos das injúrias.

As manifestações do LES podem ser gerais como fadiga, febre, perda de peso, linfoadenopatia e as mais específicas, de acordo com os comprometimentos localizados em órgãos ou sistemas. Todos os sistemas orgânicos podem ser afetados. As manifestações de pele e mucosas, músculo-esqueléticas, renais, pulmonares, cardiovasculares, hematológicas e do sistema nervoso ocorrem com maior freqüência e com maior gravidade ${ }^{(5)}$.

A terapêutica individualizada depende do comprometimento de órgãos e sistemas, assim como da gravidade de cada caso. Em geral, abrange dois tipos de tratamento: o não medicamentoso e o medicamentoso.

No primeiro inclui-se educação em saúde: orientações sobre a doença e seu tratamento, atividade física e repouso, proteção contra a luz solar, alimentação balanceada, acompanhamento ginecológico e de planejamento familiar, controle da hipertensão, prevenção de infecção e de complicações, formas de amenizar sinais e sintomas que comprometem a auto-imagem e auto-estima e outras, além de apoio psicológico e social.

Segundo o Consenso Brasileiro Para o Tratamento do Lúpus Eritematoso Sistêmico ${ }^{(4)}$, o tratamento medicamentoso tem a finalidade de reduzir a atividade inflamatória da doença, controlar sinais e sintomas e reduzir complicações. A corticoterapia prolongada (glicocorticóides, preferencialmente a prednisona) e antimaláricos (preferencialmente difosfato de cloroquina ou sulfato de hidroxicloroquina) são as drogas mais utilizadas. Em situações de maior gravidade, como na presença de comprometimento renal e lesões cutâneas, a terapia pode ser mais agressiva com elevadas doses de corticosteróide, muitas vezes associado a imunossupressor, denominada de pulsoterapia ${ }^{(4)}$

O presente estudo teve como objetivo relatar a experiência de docentes e alunos na assistência a pessoas adultas, lúpicas, submetidas a pulsoterapia, especialmente mulheres, estabelecendo o rol de diagnósticos de enfermagem (DE) específicos nestes casos e a assistência de enfermagem.

\section{CONHECENDO SOBRE A PULSOTERAPIA}

A pulsoterapia consiste na terapia ministrada por via endovenosa, durante curto período de tempo, em sessões, com doses elevadas de corticosteróide, podendo haver associação com imunossupressor antineoplásico. Sua finalidade é controlar rapidamente o processo inflamatório das doenças difusas do tecido conjuntivo. Esta terapia está indicada no tratamento de uma ampla variedade de doenças crônicas, em crianças e adultos.

No LES é utilizada quando há comprometimento multivisceral, lesões em órgãos nobres como rins, pulmões, cérebro e lesões cutâneas ${ }^{(4,6)}$.

Cada sessão de pulsoterapia em adulto consiste na infusão durante duas a três horas, de uma solução endovenosa prescrita pelo médico, composta de $100 \mathrm{ml}$ Soro Fisiológico 0,9\% ou Soro Glicosado a 5\%, contendo Metilprednisolona em doses que variam de 500 a $1000 \mathrm{mg}$; freqüentemente com adição de Ciclofosfamida de 500 a 1000 mg por metro quadrado ou $750 \mathrm{mg}$ por metro quadrado de superfície corporal; antiemético, que pode ser Metoclopramida $10 \mathrm{mg}$ ou Ondansetrona $4 \mathrm{a} 8 \mathrm{mg}^{(6)}$. A Ondansetrona, por ter indicação específica no controle de náuseas e vômitos induzidos por quimioterapia citotóxica emetogênica, tem sido bastante prescrito às pacientes que assistimos.

A apresentação da Metilprednisolona é em ampolas de $40 \mathrm{mg}, 125 \mathrm{mg}$, $500 \mathrm{mg}$ e $1000 \mathrm{mg}$; Ciclofosfamida encontra-se em ampolas de $50 \mathrm{mg}$ ou 200 $\mathrm{mg}$; Metoclopramida, em ampolas de $2 \mathrm{ml}$ com $10 \mathrm{mg}$; Ondansetrona, em ampolas de $2 \mathrm{ml}$ com $4 \mathrm{mg}$ e de $4 \mathrm{ml}$ com $8 \mathrm{mg}^{(7)}$.

Destacamos que as reações adversas em adultos e crianças são freqüentes, assim como os efeitos colaterais relacionados a essa terapia. $A$ experiência em clínica leva-nos a concordar com Gonçalves et al(8), os quais em um estudo para determinar a freqüência e severidade dos efeitos adversos durante a pulsoterapia com infusão de metilprednisolona e ciclofosfamida em 70 crianças e adolescentes portadoras de doenças reumáticas, encontraram que $70,3 \%$ apresentaram reações adversas, sendo as mais freqüentes: gosto amargo (48\%), hiperemia facial (40\%), vômitos ( $78,3 \%$ ) e náuseas $(48,3 \%)$. Nesse estudo os efeitos, embora controlados durante a infusão, foram limitantes para a terapia.

As pessoas submetidas a esta modalidade de tratamento podem apresentar hipertensão arterial sistêmica, retenção de água e sódio, perda de cálcio e potássio, náuseas, vômitos, hiperglicemia e risco elevado de adquirir infecção devido à imunossupressão. Por isso, podem necessitar de terapia adicional com anti-hipertensivos, antieméticos, insulinoterapia, reposição de eletrólitos e assistência de enfermagem direcionada a essas reações adversas, inclusive com indicação de isolamento protetor.

\section{APLICANDO A METODOLOGIA DA ASSISTÊNCIA NO ENSINO}

Constatamos que os dados epidemiológicos referentes ao LES, descritos na literatura, estão em consonância com a realidade encontrada nos campos de prática. Por isso, conferem coerência à proposta da disciplina de Enfermagem Clínica de colocar o aluno cada vez mais próximo de uma realidade que requer ações efetivas de assistência, com relação de qualidade entre profissional de saúde/cliente e com atenção individualizada e ajustada às necessidades e possibilidades do paciente. Assim, estabelecemos, como metodologia de ensino, a aplicação do Processo de Enfermagem para esses casos.

Os longos períodos de internação a que tais pessoas estão propensas que, em muitos casos, causam dependência física e emocional, favorecem a aplicação de um instrumento de sistematização da assistência de enfermagem, que permita uma visão global do ser humano assistido.

Após as fases de levantamento dos dados (anamnese, exame físico e revisão de prontuário) das pacientes assistidas, estabelecemos um rol de Diagnósticos de Enfermagem (DE) comuns à maioria delas.

\section{LISTANDO DIAGNÓSTICOS DE ENFERMAGEM}

Alguns DE são comuns aos pacientes submetidos a essa terapia. Mas podem divergir de um paciente para outro, pois são elaborados a partir das características definidoras, as quais se originam de dados subjetivos e objetivos coletados no histórico e exame físico de cada paciente. Os DE mais evidenciados nas pacientes submetidos à pulsoterapia e assistidos 
durante as atividades práticas e de estágio foram:

1) Adaptação prejudicada, relacionada à incapacidade que exige mudança no estilo de vida, sistemas de apoio inadequados, negação da doença, agressão à auto-estima e doença que ameaça a vida;

2) Déficit de conhecimento (necessidade de aprendizagem), relacionado à falta de informação sobre a doença, interpretação incorreta das informações e limitações cognitivas;

3) Déficit de lazer, relacionado à imobilidade, hospitalizações freqüentes, restrições do tratamento, fadiga e indisposição;

4) Déficit do volume de líquidos, relacionado à falha nos mecanismos reguladores (supra-renais, hiperglicemia, vômitos);

5) Déficit no auto-cuidado (alimentação, banho, higiene, vestir-se/ arrumar-se, uso de vaso sanitário), relacionado a prejuízo músculoesquelético, diminuição da força e da capacidade de suportar amplitude de movimentos;

6) Desempenho de papel alterado, relacionado à doença crônica, mudança na capacidade física, hospitalizações freqüentes e prolongadas;

7) Distúrbio da auto-estima/auto-imagem, relacionado às mudanças biofísicas (lesões na pele, perda de cabelos, emagrecimento) e psicossociais (mudanças no estilo de vida, dependência dos outros para auxílio);

8) Dor aguda relacionada a agentes causadores de lesão (processo inflamatório disseminado e trauma tissular);

9) Excesso do volume de líquidos relacionado a mecanismo regulador comprometido e terapia com corticosteróides;

10) Fadiga, relacionada à química do corpo alterada, demandas psicológicas e emocionais aumentadas e estados de desconforto.

11) Impotência (nível pessoal), relacionada à natureza crônica da doença, falta de controle sobre resultados, incapacidade de cuidar de si mesmo e depressão;

12) Integridade da pele prejudicada, relacionada a fatores externos (punção venosa) e internos (medicamentos, processo inflamatório, déficit imunológico);

13) Integridade tissular prejudicada, relacionada à circulação alterada, déficit/excesso de líquidos, alterações nutricionais e irritantes químicos e mecânicos:

14) Interação social prejudicada relacionada a isolamento terapêutico e mobilidade física limitada;

15) Intolerância à atividade relacionada à fraqueza generalizada, dor e repouso ao leito;

16) Manutenção da saúde alterada, relacionada à falta de recursos materiais e estratégias ineficazes de resolução familiar;

17) Medo relacionado à ameaça de morte percebida ou real;

18) Mobilidade física prejudicada, relacionada à terapia restritiva, desconforto e intolerância à atividade;

19) Mucosa oral alterada relacionada às condições patológicas;

20) Nutrição alterada: ingestão menor do que as necessidades corporais, relacionada à incapacidade para ingerirnutrientes adequados (náuseal vômitos) e restrições alimentares terapêuticas;

21) Perfusão tissular alterada, relacionada à redução do volume de sangue, redução do fluxo sangüíneo renal, resistência vascular sistêmica alterada e aumento da atividade simpática (hipertensão);

22) Proteção alterada, relacionada a perfis sangüíneos anormais (anemia), terapias com fármacos (corticosteróides, imunossupressores) e doença inflamatória crônica;

23) Risco para infecção, relacionado a defesas primárias e secundárias inadequadas (lesões de pele, acesso venoso, resposta inflamatória suprimida) e imunossupressão (doença crônica, agentes farmacêuticos, níveis elevados de glicose, procedimentos invasivos e desnutrição);

24) Risco para senso-percepção alterada, relacionado à alteração da química endógena (desequilibrio entre níveis de glicose/insulina e eletrólitos).

\section{PRESTANDO ASSISTÊNCIA DE ENFERMAGEM}

Didaticamente, a implementação da assistência individualizada requer o cumprimento antecipado, em sequiência, da análise cuidadosa dos diagnósticos de enfermagem levantados, definição de objetivos claros no plano de assistência contendo cuidados de enfermagem. Seguindo o exercício acadêmico, os cuidados de enfermagem elencados são posteriormente transformados em prescrição de enfermagem, para que ocorra de fato a individualização da assistência.

Os objetivos contidos no plano de assistência da maioria dessas pacientes foram:

- Acompanhar a evolução clínica;

- Prevenir complicações e infecções;

- Restabelecer e manter o equilíbrio hidroeletrolítico;

- Restabelecer e manter a integridade cutânea, mucosa e tissular;

- Promover apoio emocional, social e espiritual;

- Proporcionar conforto, higiene e segurança;

- Realizar educação em saúde (para paciente e acompanhante).

Apresentamos a seguir os cuidados de enfermagem que, além de prioritários, são especificamente dirigidos/relacionados à pulsoterapia e que foram aplicados na maioria das pacientes assistidas.

- Manter em isolamento protetor;

- Avaliar nível de consciência;

- Avaliar estado geral, nutricional e nível de dependência;

- Realizar exame físico dirigido antes, durante e após cada sessão;

- Realizar punção com cateter de média duração em veia calibrosa (fixação com fita hipoalergênica);

- Preparar e administrar a pulsoterapia prescrita em bomba de infusão;

- Avaliar freqüentemente o local do acesso venoso quanto à permeabilidade, infiltração, sinais flogísticos e condições da fixação;

- Promover repouso (relativo ou absoluto considerando a gravidade e tolerância de cada paciente);

- Proporcionar condições para sono e repouso: ambiente silencioso e com pouca luz;

- Avaliar a ocorrência de reações adversas (boca amarga, náuseas e vômitos, hipertensão, hiperglicemia);

Na presença de episódios de náuseas e vômitos:

- Reduzir temporariamente o gotejamento da infusão terapêutica;

- Oferecer recipiente durante episódios de vômitos ou abrir a sonda nasogástrica (se for o caso);

- Conversar com a paciente encorajando o enfrentamento da situação;

- Suspender temporariamente a alimentação;

- Interagir com a nutricionista para reformulação da dieta, se for o caso;

- Realizar higiene oral com solução de bicarbonato de sódio (ou outra não alcoólica) após o episódio de vômito;

- Preparar e administrar soluções endovenosas prescritas para reposição de eletrólitos;

- Registrar no prontuário como intercorrência.

- Controlar sinais vitais antes durante e após cada sessão;

- Realizar curva térmica;

- Fazer controle de pressão arterial;

- Fazer controle de diurese;

- Fazer balanço hídrico rigorosamente;

- Fazer controle de peso;

- Fazer controle de edemas;

- Avaliar resultados de exames laboratoriais - de controle/terapêuticos (bioquímica: sódio, potássio, cálcio, uréia, creatinina, urina I, urocultura, hemograma e glicemia, outros) e de diagnóstico (raios- $X$, tomografia computadorizada, ultrassonografia, outros); 
- Realizar glicemia capilar durante e após a pulsoterapia;

- Atentar para sinais e sintomas de hiperglicemia;

- Administrar insulina prescrita, se for o caso;

- Interagir com a nutricionista na adequação/reformulação da dieta a ser mantida após as sessões;

- Oferecer líquidos conforme a tolerância pela via de alimentação estabelecida a cada caso;

- Incentivar aceitação da alimentação pela via estabelecida;

- Solicitar avaliação/parecer/acompanhamento do assistente social (considerar dados do histórico);

- Solicitar avaliação/ parecer/acompanhamento do psicólogo (considerar dados do histórico);

- Solicitar avaliação/parecer/acompanhamento do líder espiritual (considerar dados do histórico);

- Realizar higiene corporal (considerar nível de dependência) com sabonete neutro;

- Realizar higiene oral com creme dental / solução bicarbonatada;

- Conversar com a paciente durante a assistência, valorizando suas queixas e permitindo que ela participe de seus cuidados;

- Incentivar e prover condições para a realização de atividades lúdicas (conforme preferência e condição clínica), como leituras, trabalhos manuais, música e outras;

- Orientar paciente e acompanhante durante a hospitalização quanto a: patologia (LES), esquema terapêutico e nutricional, importância da adesão e manutenção de tratamento, prevenção de complicações, uso de filtro solar, evitar contato com pessoas portadoras de infecção, acompanhamento ginecológico, planejamento familiar e adaptações apropriadas, considerando estilo de vida de cada paciente a partir da sua história;

- Orientar repouso e compressas mornas nas articulações antes do banho morno, se apresentar artralgia matinal;

- Orientar mudança de decúbito, movimentação ativa e passiva das articulações (considerado o tipo de repouso apropriado);

- Interagir com acompanhante e pessoas próximas, orientando a necessidade de interação social, participação em eventos sociais significativos para a paciente (considerar dados coletados histórico);

- Transmitir informações positivas sobre os filhos (geralmente são menores), fatos e pessoas significativas à paciente;

- Incentivar o auto-cuidado adequando preferências e limitações como 0 uso de shampoo e sabonete neutros, evitar tinturas nos cabelos e produtos desconhecidos sobre a pele, usar maquiagem hipoalergênica, creme hidratante a base de água para o corpo, manter unhas limpas e aparadas, realizar atividade física moderada, respeitando a tolerância, sugerir formas de aliviar o estresse (considerar dados sócio econômico contidos no histórico);

- Construir plano individualizado para a alta.

\section{AVALIANDO A ASSISTÊNCIA E REGISTRANDO A EVOLUÇÃO}

A avaliação da assistência é caminho, caracterizado pela reavaliação clínica e contextualização, que visa o registro da evolução. Este processo é descrito diariamente como parte integrante do relatório de enfermagem, momento em que 0 acadêmico estabelece a co-relação entre a assistência prestada e alterações identificadas (o que melhorou, piorou, acrescentou e o que se manteve).

\section{AVALIANDO A APRENDIZAGEM}

Com finalidade de avaliação da aprendizagem, os acadêmicos de enfermagem, em grupos de três a quatro elementos, cumprem a tarefa de elaborar e apresentar um estudo de caso, com enfoque prioritário na metodologia da assistência de enfermagem, mas que contempla o estudo das patologias, medicamentos administrados, avaliação e interpretação de exames que a paciente realizou e protocolos de cuidados de enfermagem, utilizando-se de referências bibliográficas atualizadas.

Os critérios de avaliação incluem aspectos afetivos, cognitivos e psicomotores e também a abrangência bio-psico-sócio-espiritual da assistência prestada.

\section{CONSIDERAÇÕES FINAIS}

O desenvolvimento da disciplina de enfermagem clínica, em condições reais de trabalho, com aplicação do Processo de Enfermagem utilizado como estratégia metodológica para assistir o paciente e ensinar os acadêmicos, tem se revelado muito satisfatório. No cuidado a pacientes submetidas a pulsoterapia, tem sido possivel prestar assistência sistematizada, individualizada e integral, o que mostra oportunidades singulares para correlação teórico-prática, com a aplicação de conhecimentos básicos da área de formação acadêmica (anatomia, fiosiologia, imunologia, farmacologia, biologia, psicologia, semiologia, semiotécnica, epidemiologia, saúde coletiva, ética, didática aplicada e outras), contextualização do processo saúde-doença e focalização ampliada para além do biológico, abrangendo aspectos emocionais, sociais e espirituais e oportunidade para o acadêmico avaliar as diversas nuanças que interferem nas condições de assistência oferecidas pelos serviços públicos de saúde.

Durante todo o tempo, tem sido evidente a escassez de bibliografias específicas sobre este tema, que em sua maioria estão disponíveis e/ou atualizadas apenas nos meios eletrônicos e freqüentemente são dirigidas à área médica, nunca à enfermagem ou aos processos de cuidar.

Desejamos desta forma, estar contribuindo para a qualidade da assistência, fornecendo material de consulta a equipe de enfermagem e acadêmicos que vivem experiências similares.

\section{REFERÊNCIAS}

1. Robazzi MLCC, Carvalho EC, Mendes MMR, Veiga EV Diagnósticos de enfermagem: atribuição feita por graduandos de enfermagem a pacientes internados com alterações neurológicas. Rev Latino-am Enfermagem 1998;6(2):37-46.

2. Iyer PW, Taptich BJ, Bernochi-Losey D. Processo e Diagnóstico em Enfermagem. Porto Alegre (RS): Artes Médicas; 1993.

3. Bonfá ED. Lúpus Eritematoso Sistêmico (LES). (citado em: 12 abr 2002). Disponível em: URL: http://www.usp.br/medicina/ departamento/reumatologia/lupus

4. Sato El. Consenso Brasileiro para o Tratamento do Lúpus Eritematoso Sistêmico (LES). Rev Bras Reumatol 2002;42(6):35561.

5. Sato El. Lupus Eritematoso Sistêmico. Sociedade Brasileira de
Reumatologia. (citado em: 9 jul 2004). Disponível em: URL: http://www.reumatologia.com.br/doe6.htm

6. Arruda A. Pulsoterapia: pulsoterapia com corticóide e pulsoterapia com ciclofosfamida (citado em: 20 jun 2004). Disponível em: URL: http://www.geocities.com/adalbertoarruda/medicos.htm

7. Jornal Brasileiro de Medicina Publicações Científicas. Dicionário de especialidades farmacêuticas - DEF. Rio de Janeiro (RJ): JBMPC; 2002.

8. Gonçalves M, Terreri MTRA, Len CA, Hilario MOE. Efeitos adversos durante a infusão de metilprednisolona e ciclofosfamida em crianças com doenças reumáticas. (citado em: 9 jul 2004). Disponível em: URL: http://www.revbrasreumatol.com.br/ edicoes/vol43_02/43_2_84.htm 